Revista Arbitrada Interdisciplinaria KOINONIA

Año V. Vol V. N`9. Enero - Junio 2020

Hecho el depósito de Ley: FA2016000010

ISSN: 2542-3088

FUNDACIÓN KOINONIA (F.K). Santa Ana de Coro. Venezuela.

Javier Valverde-Bastidas; Mario Cesén-Arteaqa; Edqar Sarmiento-Boria

http://dx.doi.org/10.35381/r.k.v5i9.657

\title{
Restauración y conservación digital de fósiles mediante escaneado 3D y la reproducción con prototipado rápido
}

\section{Restoration and digital preservation of fossils through 3D scanning and reproduction with rapid prototyping}

\author{
Javier Valverde-Bastidas \\ javier.valverde@epn.edu.ec \\ Escuela Politécnica Nacional, Quito \\ Ecuador \\ https://orcid.org/0000-0002-8367-6844 \\ Mario Cesén-Arteaga \\ mario.cesen@epn.edu.ec \\ Escuela Politécnica Nacional, Quito \\ Ecuador \\ https://orcid.org/0000-0002-6778-9945 \\ Edgar Sarmiento-Borja \\ edgar.sarmiento@epn.edu.ec \\ Escuela Politécnica Nacional, Quito \\ Ecuador \\ https://orcid.org/0000-0003-0344-2974
}

Recibido: 12 de noviembre de 2019

Revisado: 30 de noviembre de 2019

Aprobado: 20 de diciembre de 2019

Publicado: 20 de enero de 2020

\section{RESUMEN}

En el caso de elementos delicados como son los fósiles, el uso del escáner es el más adecuado, por cuanto éste maneja una tecnología que permite recopilar la información de forma digital con el propósito de: levantamiento de información, modelamiento o restauración o inclusive luego del modelamiento, se puede realizar ensamblajes de elementos individuales para duplicar elementos o recrear cuerpos completos. Los resultados de la aplicación desarrollada en este trabajo, tienen aproximaciones muy 


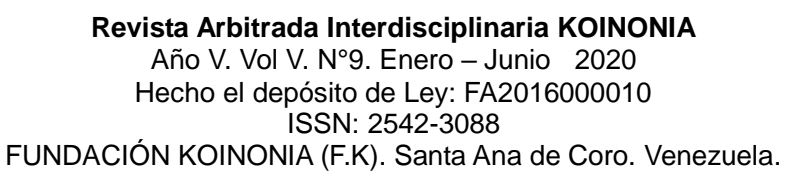

Javier Valverde-Bastidas; Mario Cesén-Arteaqa; Edqar Sarmiento-Boria

cercanas a las reales, evidenciándose la efectividad de la metodología desarrollada. Posteriormente y una vez obtenido el modelo 3D, se procede a realizar las operaciones necesarias para la reproducción del fósil reconstruido utilizando equipo de prototipado rápido; para lo cual se emplea software adecuado que permita realizar estos procedimientos y, de esta manera, realizar la integración completa desde escaneo 3D hasta la obtención del elemento final de forma digital o física.

Descriptores: Fósil; ingeniería de la producción; robótica; reconocimiento de formas. (Palabras tomadas del Tesauro UNESCO).

\begin{abstract}
In the case of delicate elements such as fossils, the use of the scanner is the most appropriate, since it uses a technology that allows the collection of information digitally with the purpose of: information gathering, modeling or restoration or even, after modeling, it is possible that individual element assemblies can be made to duplicate elements or recreate whole bodies. The results of the application developed in this work, have approximations very close to the real ones, evidencing the effectiveness of the implemented methodology. Subsequently and once the 3D model is obtained, the necessary operations for the reproduction of the reconstructed fossil are carried out using rapid prototyping equipment; for which suitable software is used to allow these procedures to be executed and, in this way, to develop the complete integration from 3D scanning to obtaining the final element in digital or physical form.
\end{abstract}

Descriptors: Fossils; production engineering; robotics; pattern recognition. (Words taken from the UNESCO Thesaurus).

\title{
INTRODUCCIÓN
}

La ingeniería inversa mediante el escaneado 3D de este trabajo se emplea para, a partir de un objeto material y empleando ciertos medios, obtener una malla o nube de puntos en tres dimensiones del mismo, con el fin de tratar esa malla posteriormente para luego realizar una reingeniería, un rediseño o directamente para volver a fabricar el objeto además que la reconstrucción 3D permite conocer aspectos geométricos de los objetos de los cuales no se tienen referencias y que son muy complicados obtenerlos comúnmente. 
El reciente avance en la ingeniería inversa, ha elevado a este tipo de tecnología, a una de las principales metodologías utilizadas por muchas industrias, tales como: la industria aeroespacial, automotriz, electrónica de consumo, dispositivos médicos, equipos deportivos, juguetes y joyas, aplicándose además la ingeniería inversa también se aplica en ciencia forense e investigaciones de accidentes.

La ingeniería inversa busca obtener información acerca de un objeto o sistema de referencia, dicha información es obtenida bajo un proceso de análisis, como por ejemplo el análisis dimensional; por lo que la información obtenida debe ser específica y siguiendo procedimientos que permitan caracterizar al objeto y la información registrada, sea la ayuda para la obtención de un modelo tridimensional a partir de un objeto inicial, el mismo que puede ser virtual o físico, al cual se le puede dar varias utilidades posteriores según sea la necesidad requerida. (Wang, 2010).

\section{Importancia del problema}

Se puede utilizar el escaneo 3D para realizar restauración de piezas u objetos, si se tiene cierta información de la parte a restaurar, se pueden manipular dicha información para completar o reconstruir, mediante la unión de mallas en software y de esta manera obtener una pieza completa restaurada digitalmente.

\section{Caso "Fósil de mastodonte"}

En el Laboratorio de Paleontología de la Escuela Politécnica Nacional, se tiene una variedad de fósiles que normalmente les tienen que hacer restauración o unir partes que se encuentran separadas, como es el caso del fósil del fémur de un mastodonte en el que la mayoría del hueso se encuentra completo, pero una parte se encuentra separado (ver figura 1). Entonces se puede proceder a escanear ambas partes para luego realizar la restauración y reconstrucción juntando las mallas en software de ingeniería inversa y 
de esta manera obtener un archivo digital; el mismo que contiene la información de la forma del fósil y que puede ser conservado como información valiosa de respaldo para el laboratorio. También con esa información se puede crear prototipos del fósil original pero ya reconstruido y completo

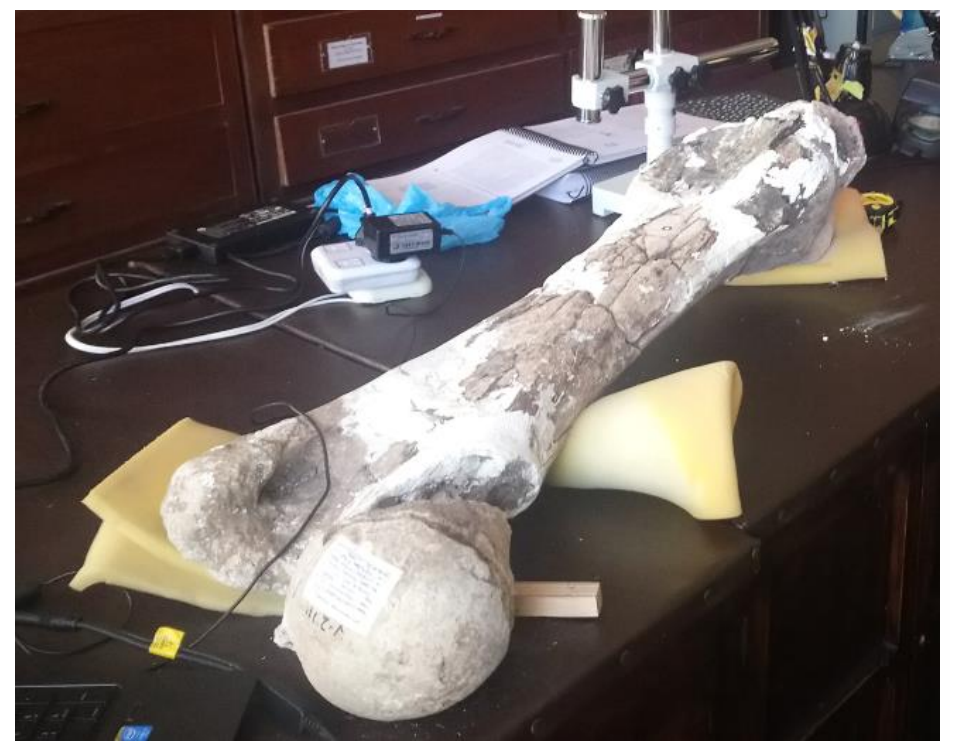

Figura 1. Fósil de mastodonte "fémur".

Fuente: Autores (2019).

Desde lo planteado, la investigación se trazó desarrollar el objetivo principal de aplicar la metodología inversa para la restauración y conservación digital de fósiles mediante escaneado 3D y la reproducción física con prototipado rápido.

\section{Aspectos teóricos referenciales}

\section{Escáner 3D}

Un escáner 3D es un dispositivo que analiza un objeto o una escena para reunir datos de su forma y ocasionalmente su color (ver figura 2). La información obtenida se puede usar para construir modelos digitales tridimensionales que se utilizan en una amplia variedad 
de aplicaciones. Desarrollados inicialmente en aplicaciones industriales (metrología y automóvil), han encontrado un vasto campo de aplicación en actividades como la arqueología, arquitectura, ingeniería y entretenimiento (en la producción de películas y videojuegos). [Ceniceros, 2017]. Al realizar un nuevo diseño mediante técnicas de ingeniería inversa con escáner 3D, el proceso se basa en utilizar para ese diseño superficies físicas ya existentes.

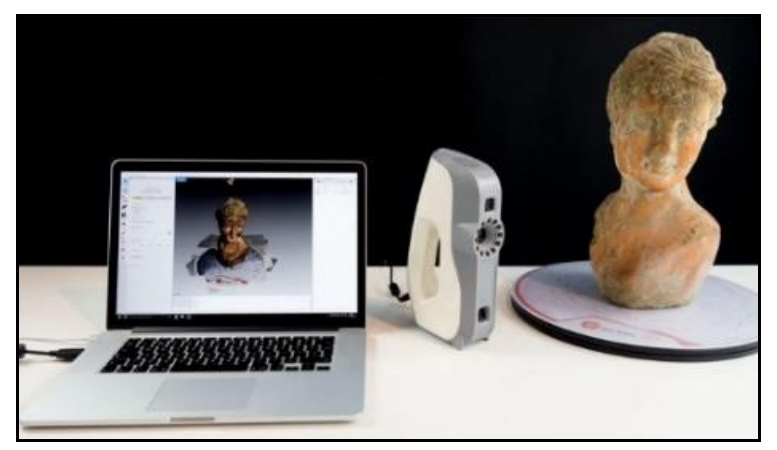

Figura 2. Escáner 3D.

Fuente:Sánchez (2017).

El primer paso en este tipo de procesos, es la digitalización de la superficie, que se realiza mediante un escáner 3D. Un escáner 3D captura puntos de la superficie de un objeto y construye lo que se denomina una nube de puntos. A partir de esta nube de puntos, con la ayuda del software del escáner se genera una superficie poligonal que consiste en una malla formada por triángulos, para seguidamente realizar una restauración virtual en base a la información anterior, mediante procedimientos y herramientas de un software adecuado para estas operaciones (ver figura 3). Luego dependerá de la aplicación final que se tenga para llegar a completar las actividades de la ingeniería inversa. [Ceniceros, 2017] 
Revista Arbitrada Interdisciplinaria KOINONIA

Año V. Vol V. N9. Enero - Junio 2020

Hecho el depósito de Ley: FA2016000010

ISSN: 2542-3088

FUNDACIÓN KOINONIA (F.K). Santa Ana de Coro. Venezuela.

Javier Valverde-Bastidas; Mario Cesén-Arteaqa; Edqar Sarmiento-Boria

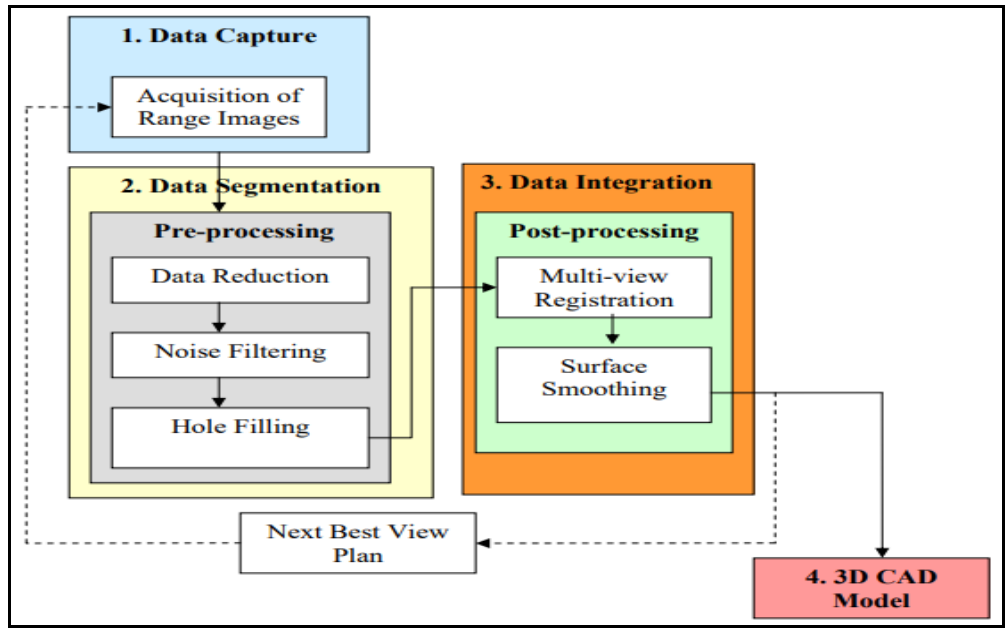

Figura 3. Secuencia de pasos para reconstrucción de un modelo mediante ingeniería inversa con escáner 3D.

Fuente:Ngozi Sherry Ali, 2005

\section{Tipos de escáner 3D}

Se puede realizar una clasificación general de dos tipos de escáner 3D (ver figura 4):

Escáner 3D por contacto: Éste tipo de escáner emplea una sonda o palpador con el que se recorre la superficie de la pieza. El palpador posiciona en el espacio tridimensional cada punto del objeto con respecto a un origen de máquina dando sus coordenadas absolutas X, Y, Z. [Martínez, 2012].

Escáner 3D sin contacto: Al contrario que el anterior tipo, este escáner no necesita mantener un contacto físico con el objeto para generar su geometría. A su vez se engloban en dos grandes grupos:

- Activos: Este tipo de escáner emite un tipo determinado de onda que es reflejada en el objeto y los sensores de los que está dispuesto el escáner reciben la señal, como por ejemplo: escáner de tiempo de vuelo, escáner de triangulación, holografía conoscópica, escáner de luz estructurada, escáner de luz modulada, etc.

- Pasivos: Es el método más económico para realizar un escaneo, utilizan radiación 
Revista Arbitrada Interdisciplinaria KOINONIA

Año V. Vol V. N9. Enero - Junio 2020

Hecho el depósito de Ley: FA2016000010

ISSN: 2542-3088

FUNDACIÓN KOINONIA (F.K). Santa Ana de Coro. Venezuela.

Javier Valverde-Bastidas; Mario Cesén-Arteaqa; Edgar Sarmiento-Boria

e impulsos del ambiente, como por ejemplo: escáner de silueta, escáner estereoscópico, escáner de fotogrametría, etc. [Martínez, 2012]

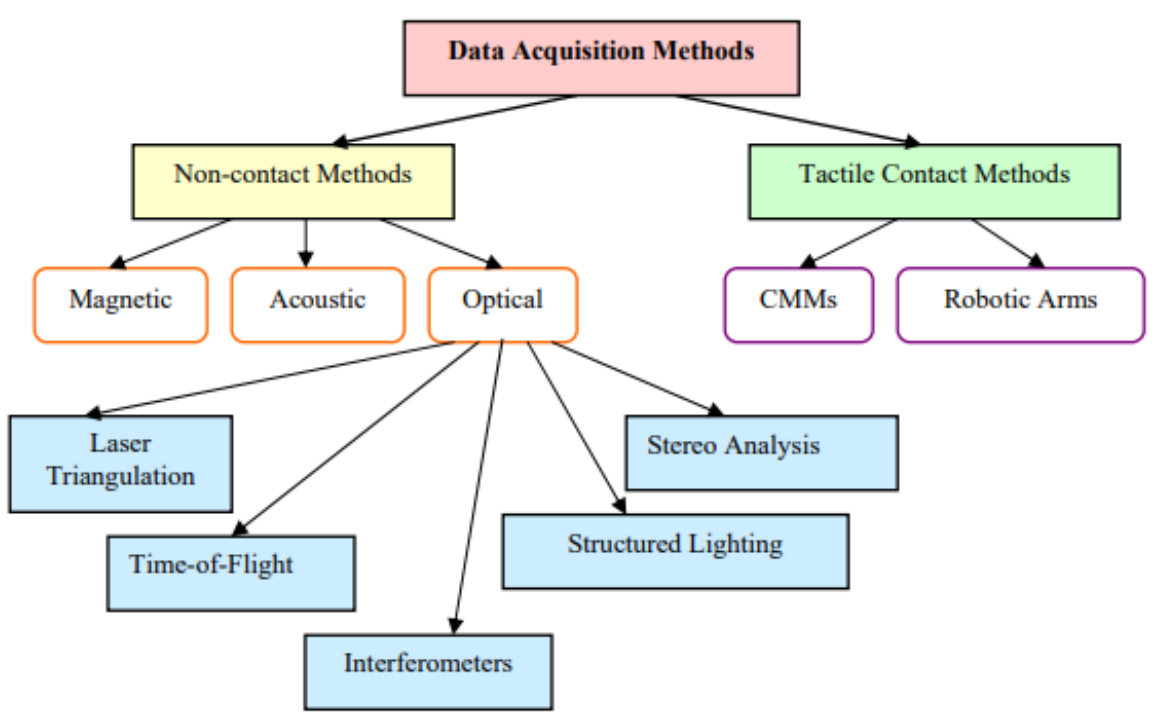

Figura 4. Métodos para adquisición de información mediante escaneo 3D de objetos.

Fuente:Ngozi Sherry Ali, 2005

\section{METODOLOGÍA}

\section{Características de los equipos y software}

Para la realización del presente trabajo se toma en cuenta los equipos y el software que se poseen en el Laboratorio de Investigación, Desarrollo e Innovación en Nuevas Tecnologías de Diseño, Manufactura y Producción (LABINTECDIMP) de la Facultad de Ingeniería Mecánica de la Escuela Politécnica Nacional, los mismos son los siguientes:

\section{Software y equipos para escaneado 3D}

\section{VXelements}

Es una plataforma de software 3D simple, potente y totalmente integrada que funciona en sinergia completa con todas las tecnologías de digitalización 3D, es decir, con este 
Revista Arbitrada Interdisciplinaria KOINONIA

Año V. Vol V. N9. Enero - Junio 2020

Hecho el depósito de Ley: FA2016000010

ISSN: 2542-3088

FUNDACIÓN KOINONIA (F.K). Santa Ana de Coro. Venezuela.

Javier Valverde-Bastidas; Mario Cesén-Arteaqa; Edgar Sarmiento-Boria

software y el escáner, se puede obtener la información de la nube de puntos del objeto escaneado tridimensionalmente (ver figura 5). [Creaform Inc., 2014]

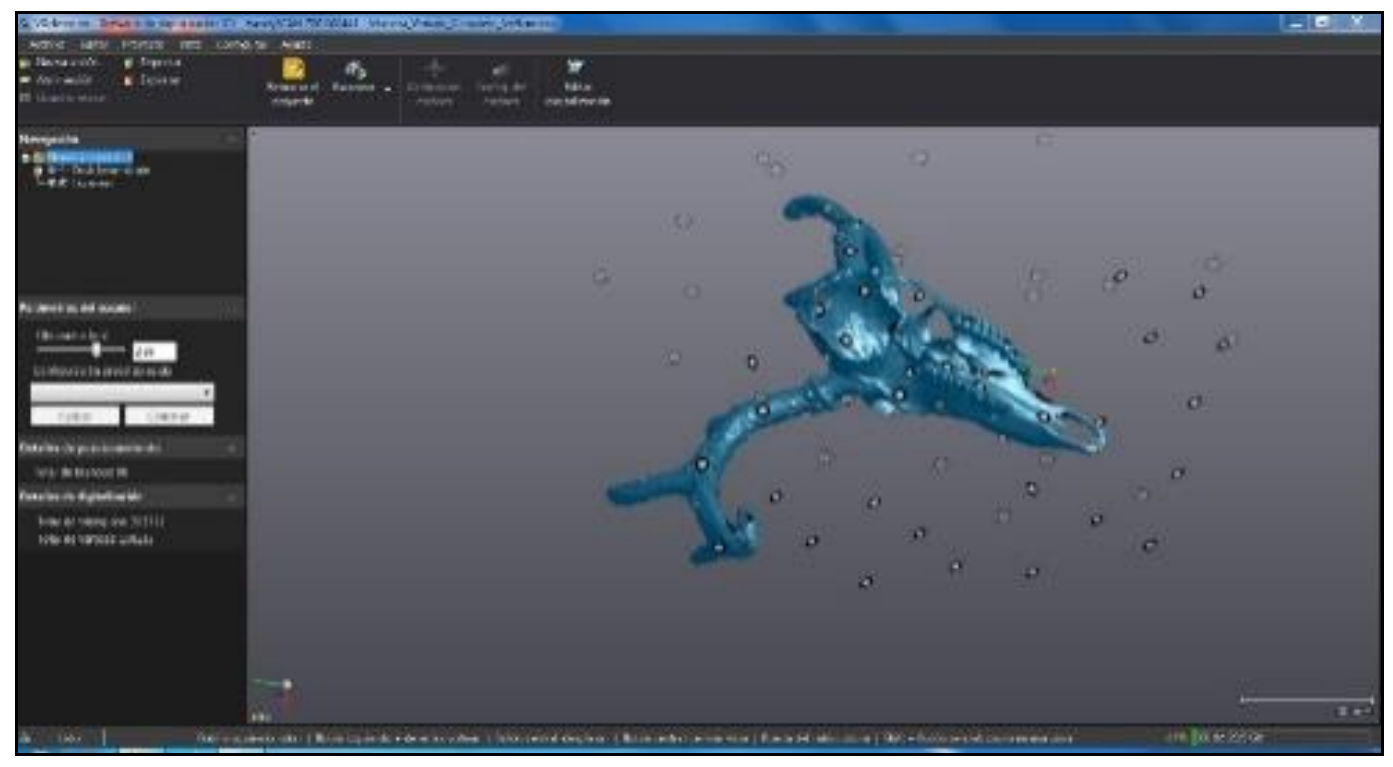

Figura 5. Ventana del software “VXelements".Fuente: Los autores, Ecuador, 2019

\section{Escáner Portátil 3D}

El escáner fijo 3D que se tiene es de la marca "3D Systems" y el modelo es el "Capture" que ofrece tecnología de escaneado 3D de luz azul, precisa y rápida dentro de una unidad portátil y muy ligera que se puede utilizar como dispositivo de mano o fijo (ver figura 6), y que además, se puede combinar fácilmente en una solución multiescáner, además que se puede añadir fácilmente un escaneado con plato giratorio sin intervención manual. [3D Systems, Inc., 2015]

Los accesorios del equipo son:

- Escáner Capture.

- Trípode o soporte.

- Mesa giratoria. 
Revista Arbitrada Interdisciplinaria KOINONIA

Año V. Vol V. Nº. Enero - Junio 2020

Hecho el depósito de Ley: FA2016000010

ISSN: 2542-3088

FUNDACIÓN KOINONIA (F.K). Santa Ana de Coro. Venezuela.

Javier Valverde-Bastidas; Mario Cesén-Arteaqa; Edqar Sarmiento-Boria

- Conector a la fuente de poder.

- Conector a la computadora.

- Laptop con software.

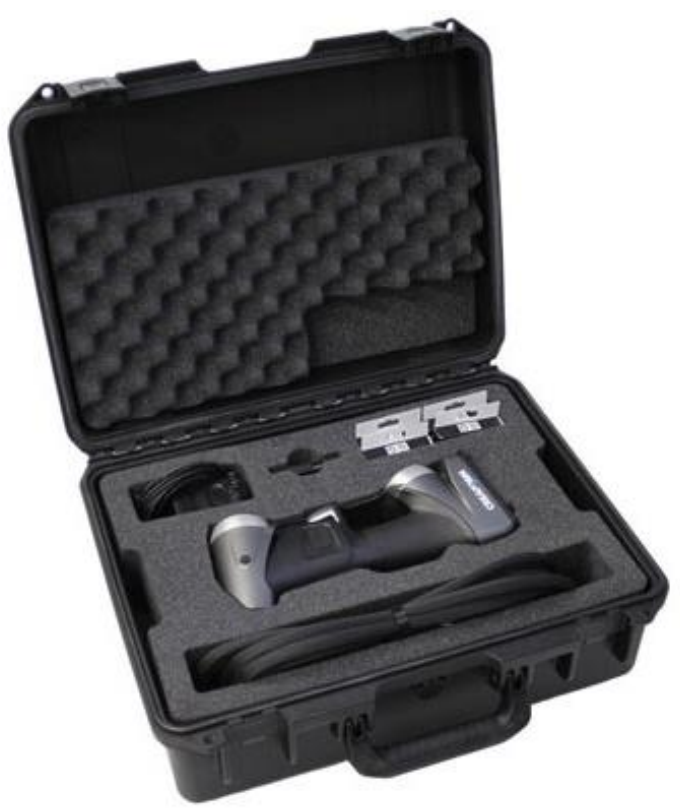

Figura 6. Escáner portátil 3D "HandyScan 700” y sus accesorios.

Fuente: Autores (2019).

Características del escáner portátil 3D "HandyScan 700".

Modelo

HandyScan 700

Peso

$0,85 \mathrm{~kg}$

Dimensiones

$122 \times 77 \times 294 \mathrm{~mm}$

Velocidad de medición

480000 mediciones/s

Área de escaneado

$275 \times 250 \mathrm{~mm}$

Fuente de luz

7 cruces láser (+1 línea extra)

Tipo de láser

II (seguro para la vista)

Resolución

$0,05 \mathrm{~mm}$ 
Precisión

Precisión volumétrica

Distancia de seguridad

Profundidad de campo

Tamaño de piezas (recomendado)

Software

Formatos de salida

Software compatible

Estándar de conexión

Rango de temperaturas de

funcionamiento

Rango de humedad de funcionamiento (sin condensación)

Fuente:Creaform Inc., 2014
Hasta $0,030 \mathrm{~mm}$

$0,020 \mathrm{~mm}+0,060 \mathrm{~mm} / \mathrm{m}$

$300 \mathrm{~mm}$

$250 \mathrm{~mm}$

$0,1-4 \mathrm{~m}$

VXelements

.dae, .fbx, .ma, .obj, .ply, .stl, .txt, .wrl, .x3d, .x3d z, .zpr

3D Systems (Geomagic ${ }^{\circledR}$ Solutions), InnovMetric Software (PolyWorks), Dassault Systems (CATIA V5 y SolidWorks), PTC (Pro/ENGINEER), Siemens (NX y Solid Edge), Autodesk (Inventor, Alias, 3ds Max, Maya, Softimage).

1 x USB 3.0

$15-40^{\circ} \mathrm{C}$ $10-90 \%$

\section{Software para post-tratamiento y reconstrucción de información de escaneado 3D} GeomagicDesign X

Es una herramienta de software inteligente para la extracción de los parámetros de diseño a partir de datos de escaneado 3D. GeomagicDesign X es una nueva e innovadora solución de software que permite a los ingenieros crear sólidos CAD 
totalmente paramétricos y superficies de forma libre a partir de datos de escaneo 3D y datos de malla de polígonos con la mejor interfaz de usuario de su clase (ver figura 7). Además de su capacidad para crear sólidos CAD paramétricos, GeomagicDesign X se diferencia de otros software de ingeniería inversa existentes, al ofrecer un proceso de diseño que utiliza los conjuntos de habilidades que los ingenieros ya poseen.

El resultado final es una solución de software que requiere una capacitación mínima, lo que reduce el tiempo necesario para procesar datos de escaneo 3D. Los usuarios reciben resultados de alta calidad que no están disponibles a través de otras opciones actuales de ingeniería inversa. GeomagicDesign $X$ mejora la funcionalidad de la tecnología de escaneo 3D y las aplicaciones CAD existentes al compartir tecnologías y procesos comunes. [3D Systems, Inc., 2015]. GeomagicDesign X cuenta con herramientas poderosas (ver figura 8), como:

- Asistente de rediseño.

- Analizador de precisión.

- Obtención de sólidos, superficies y mallas.

- Asistente de alineación.

- Las mejores operaciones de malla en su clase.

- Herramientas sofisticadas de curva/boceto.

- Sincronización de escaneo a CAD.

- Malla rápida a la superficie.

- Transferencia de datos sin problemas.

- Limpieza, curación y optimización de malla.

- Rediseño dentro de tolerancias definidas por el usuario.

- Flujos de trabajo de modelado de sólidos y superficies estándar. 


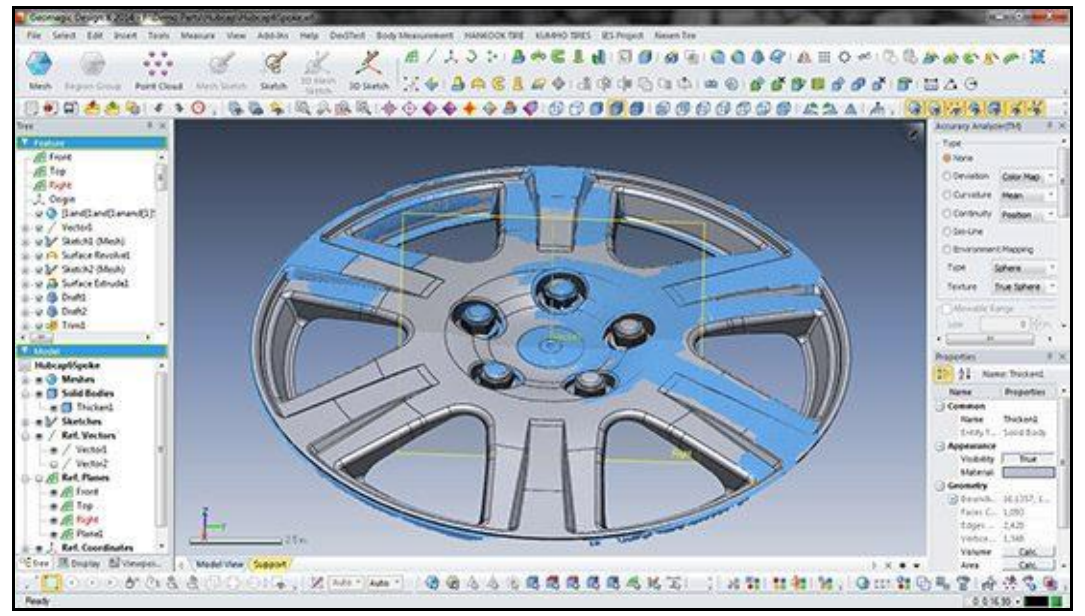

Figura 7. Software GeomagicDesign X.

Fuente:Accurex, 2017

\section{Equipo para prototipado rápido}

\section{Estación de prototipado rápido}

La estación de prototipado rápido es un equipo CNC que permite obtener prototipos o piezas normalmente de tamaños pequeños y medianos, mediante procesos de sustracción de material (ver figura 8).A diferencia de las máquinas CNC normales, estos equipos trabajan con materiales menos duros, tales como: madera, plásticos, duralón, grilón, etc., debido a que son máquinas didácticas y muy amigables de utilizar, ya que son controladas por computadoras en las cuales se carga el software de manejo de los equipos. Una estación de prototipado rápido es similar a un centro de mecanizado, pero a menor escala y con menor cantidad de herramientas para su uso.

Las estaciones de prototipado rápido que se tienen a disposición para este trabajo son de la marca "Roland" y modelo "MDX-40A" y trabajan de dos maneras, la primera mediante un software CAM propio del equipo llamado "SRP Player" y la segunda forma puede ser con cualquier CAM que genere códigos compatibles con estos equipos. Al ser un equipo CNC, estas máquinas pueden ser programadas hasta la utilización de un 
cuarto eje de programación. [Roland Inc., 2015].

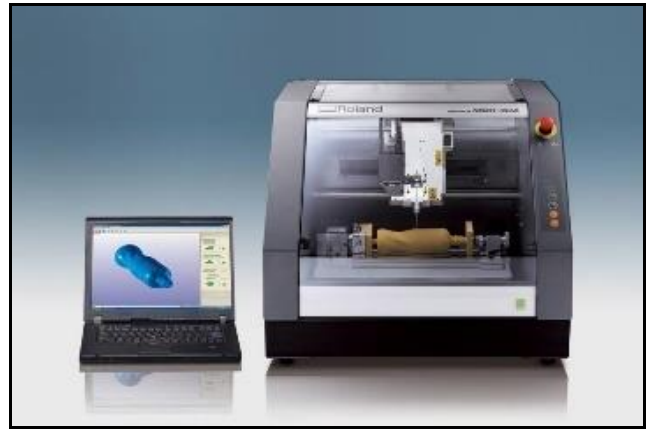

Figura 8. Estación de prototipado rápido "Roland MDX-40A" y software SRP Player.

Fuente: Roland DGA, 2019

Características de la estación de prototipado rápido "Roland MDX-40A".

Modelo de máquina

Materiales aceptables

Recorridos X, Y Z

Distancia de la punta del husillo a la mesa

Dimensión de la mesa

Peso de la pieza cargable

Motor de los ejes XYZ

Velocidad de alimentación

Resolución de software

Resolución mecánica
MDX-40

Resinas, tal como productos químicos, madera y cera de modelado (el metal no es compatible) $305(\mathrm{X}) \times 305(\mathrm{Y}) \times 105(\mathrm{Z}) \mathrm{mm}$

Máximo 123 mm

$305 \times 305 \mathrm{~mm}$

$4 \mathrm{~kg}$

Motor de movimiento gradual

Ejes $X Y: 0,1$ a $50 \mathrm{~mm} / \mathrm{s}$

Eje Z: 0,1 a $30 \mathrm{~mm} / \mathrm{s}$

Código NC: $0,001 \mathrm{~mm} /$ paso

RML-1: 0,01 mm/paso

$0,002 \mathrm{~mm} /$ paso 
Motor de husillo

Rotación del husillo

Mandril

Interfaz

Fuente de alimentación

Consumo de energía

Nivel de ruido acústico

Dimensiones totales

Peso

Temperatura de funcionamiento

Humedad de funcionamiento

Modelo de cuarto eje

Ángulo de rotación máximo

Tamaño máximo de la pieza de trabajo

cargable

Máximo grosor sostenible por la

prensa de la pieza

Peso de la pieza cargable

Velocidad de alimentación

Resolución mecánica

Dimensiones

Peso

Fuente: Roland DGA, 2019
Motor de CC (sin escobillas), máximo 100 W

4500 a 15000 rpm

Casquillo

USB

CA 100 a $240+/-10 \%$ V, 50/60 Hz, 2,1 A

Aprox. 210W

Funcionamiento sin carga: $56 \mathrm{~dB}(\mathrm{~A})$ o menos, en espera: $42 \mathrm{~dB}(\mathrm{~A})$ o menos

$669 \times 760 \times 554 \mathrm{~mm}$

$65 \mathrm{~kg}$

5 a $40^{\circ} \mathrm{C}$

35 a $80 \%$ (sin condensación)

ZCL-40

$+/-18 \times 105^{\circ}(+/-5000$ rotaciones $)$

$120 \mathrm{~mm}$ de diámetro por $270 \mathrm{~mm}$ de longitud aprox.

10 a $45 \mathrm{~mm}$

$1 \mathrm{~kg}$ (incluidas las prensas)

0,23-11,79 rpm

$0,005625 \%$ paso

$470 \times 286 \times 115 \mathrm{~mm}$

$7,5 \mathrm{~kg}$ 
Revista Arbitrada Interdisciplinaria KOINONIA

Año V. Vol V. Nº. Enero - Junio 2020

Hecho el depósito de Ley: FA2016000010

ISSN: 2542-3088

FUNDACIÓN KOINONIA (F.K). Santa Ana de Coro. Venezuela.

Javier Valverde-Bastidas; Mario Cesén-Arteaqa; Edgar Sarmiento-Boria

\section{Procedimiento de escaneado 3D, post-tratamiento y reconstrucción 3D del fósil}

La metodología para realizar la restauración del fósil de forma digital es la siguiente:

- Preparación de los objetos de referencia.- Consiste en realizar una limpieza de las partes del fósil.

- Colocación de blancos de referencias.- Consiste en colocar "targets" o blancos de posicionamiento sobre las partes a escanear, de tal forma que se tenga referencias fijas en los objetos, los cuales van a ser reconocidos por el escáner (ver figura 9).

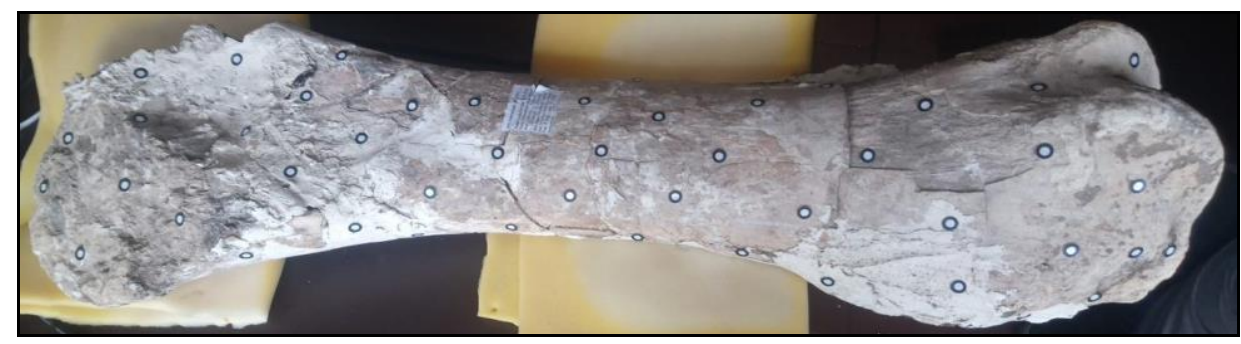

Figura 9. Preparación del fósil y colocación de "targets".

Fuente: Autores, 2019

- Instalar el escáner y el software.- Consiste en instalar todos los accesorios y el escáner a una computadora. El software utilizado es el que trae el equipo, llamado VXelements.

- Configurar en el software el escáner.- Consiste en configurar la resolución y la velocidad del obturador del escáner en base a las características del objeto de referencia (fósil).

- Escanear los objetos.- Consiste en realizar un barrido con el escáner por todas las superficies de los objetos o partes (ver figura 10). 


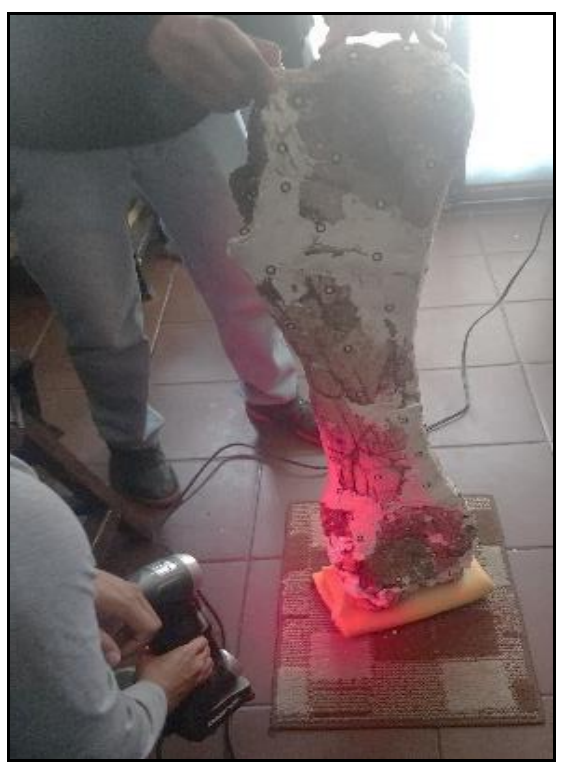

Figura 10. Escaneo del fósil y sus partes.

Fuente:Autores, 2019

Post-tratamiento: Las operaciones del post-tratamiento consisten en:

- Si se desea cambiar algunos datos sobre el archivo que contiene la información escaneada; datos tales como: "Resolución", "Optimizar la malla de la digitalización", "Reducir la malla de digitalización", "Optimización de fronteras" y "Rellenar agujeros automáticamente", se puede cambiar los datos ingresados al inicio del escaneo dependiendo la necesidad que se requiera y además se puede "Optimizar la superficie" de tal manera que se encuentre los mejores parámetros de escaneado. Una de las opciones más utilizadas es "Llenar agujeros automáticamente", ya que con esta opción, se completa cierta información faltante en las superficies del objeto escaneado que faltó leer al momento del escaneado y que depende del valor que se establezca.

- Limpieza de archivo escaneado en VXelements.- Cuando se termina la sesión de escaneado, normalmente la malla de escaneo tiene errores o excesos de 
Revista Arbitrada Interdisciplinaria KOINONIA

Año V. Vol V. Nº. Enero - Junio 2020

Hecho el depósito de Ley: FA2016000010

ISSN: 2542-3088

FUNDACIÓN KOINONIA (F.K). Santa Ana de Coro. Venezuela.

Javier Valverde-Bastidas; Mario Cesén-Arteaqa; Edgar Sarmiento-Boria

escaneos ajenos a la forma del objeto de referencia, razón por la cual se debe realizar la limpieza de esa información innecesaria conocida como parcelas aisladas o ruido de escaneo.

- Alineación de objeto escaneado en VXelements.- Cuando se escanea un objeto, normalmente el mismo se ubica en una posición distinta al origen y mucho menos el objeto se escanea alineadamente, entonces en el software VXelements tiene una opción de "Alinear con origen", Hay dos formas principales de alinear con origen en el software:

a. Alineación automática.- Realiza una aproximación a una alineación por defecto.

b. Alineación manual.- Si la alineación automática no es la que se requiere, se puede realizar de forma manual de acuerdo a como el usuario necesite

- Fusión de objetos escaneados en el software VXelements.- Si se tiene varias sesiones de escaneo de un mismo objeto se puede fusionar las mismas en el software VXelements mismo o caso contrario se puede unir mallas en formato (.stl) de un mismo objeto en el software GeomagicDesign X. Esta operación se la puede realizar de dos maneras:

a. Objetivos de mejor ajuste.- Esta herramienta alinea la sesión resaltada de la lista usando los objetivos de posicionamiento comunes de las sesiones cuando no están en la misma referencia (es decir, no escaneados usando el mismo modelo de posicionamiento de objetivo.

b. Mejor ajuste de la superficie.- Esta herramienta alinea las sesiones resaltadas de la lista usando la superficie común cuando no están en la misma referencia (es decir, no escaneados usando el mismo modelo de posicionamiento de objetivo).

- Herramientas para el post-tratamiento de información en GeomagicDesign X.- Se 
debe realizar mejoras a la malla generada en el software VXelements, para lo cual se pueden utilizar las siguientes herramientas (ver figura 11):

a. Alineación, fusión y transformación de objetos en GeomagicDesign X.- Si no se desea realizar la alineación o la fusión de varios escaneos en el software VXelements, se puede realizar dichas operaciones directamente en el software GeomagicDesign X, para lo cual existen las siguientes opciones que se encuentran en la barra de menús "ALIGNMENT"

b. En la barra de menús POLYGONS, la herramienta "MeshBuildupWizard" es automática y se obtiene mejoras en la información de escaneo.

c. También se puede hacer un curado mágico con la herramienta "HealingWizard". Cuando se selecciona esta opción, el programa automáticamente detecta los errores que tenga la información de la malla.

d. Otra de las herramientas utilizadas en el GeomagicDesign X, es "FillHoles", la cual permite rellenar agujeros que no fueron llenados en el software VXelements. La ventaja de utilizar esta herramienta, es que me permite llenar uno a uno agujeros que se encuentren presentes todavía en la malla y la posibilidad de seleccionar la forma de cómo se va a llenar los agujeros.

e. Para optimizar la malla, se utiliza la opción "OptimizeMesh", la cual sirve para aplicaciones de elementos finitos y para la operación de Auto Surface.

f. En algunas ocasiones, es importante hacer un remallado en todo el objeto, para lo cual se utiliza la herramienta "Global Remesh".

g. Y, si el objeto le falta alguna información que no se obtuvo en la fase de escaneado se puede utilizar herramienta "Rewrap", la misma que crea una malla de forma uniforme con bordes múltiples, rellenando los orificios en función de la curvatura y las formas de las características de la información de escaneo. 
h. Adicionalmente existen herramientas como: "Smart Brush" (mejora mediante herramienta de brocha o pincel), "Defeature" (elimina errores específicos con llenado de agujeros inteligente), "RemoveMarkers" (remueve marcas de blancos de posicionamiento), "Decimate" (reduce el número de triangulaciones en la malla), "Subdivide" (Aumenta el número de triangulaciones en la malla), "Smooth" (aumenta la suavidad de una malla) y también existen herramientas de edición (Split, Trim, Fix Normal, EditBoundaries, SewBoundaries, ConverttoMesh, Offset, Thicken y AddTexture).

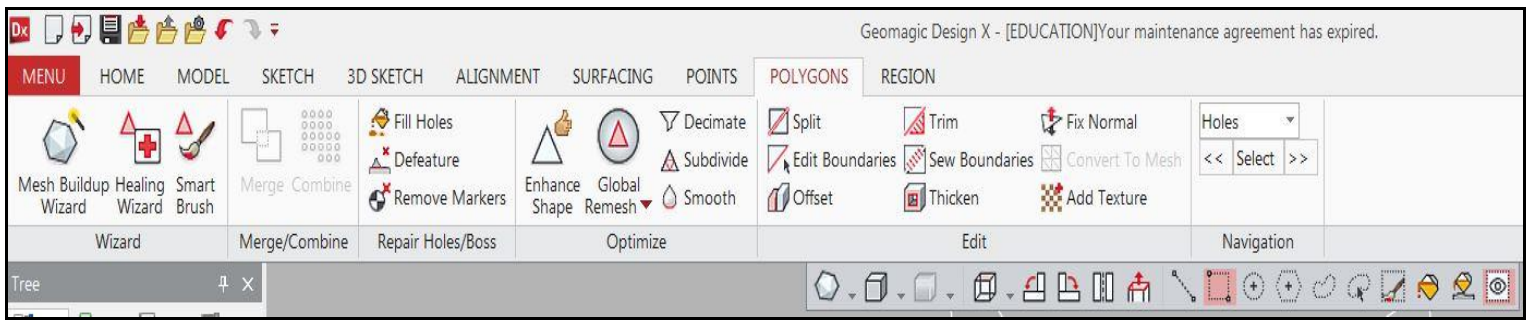

Figura 11. Herramientas de "POLYGONS" para post-tratamiento de malla.

Fuente:Autores, 2019

Reconstrucción de los objetos.- Existen tres tipos de objetos de referencia a tomar en cuenta para la realización de la ingeniería inversa mediante escaneado 3D y son los siguientes:

a. Los objetos que son de tipo orgánico, que son piezas que tienen formas muy variadas, por ejemplo esculturas, piezas arqueológicas, fósiles, etc.

b. Los objetos que tienen superficies bien definidas, es decir formas definidas por capas o superficies, por ejemplo las carcasas.

c. Los objetos que tienen formas sólidas definidas, tales como extrusiones, revoluciones, cilindros, solevaciones, toroides, esferas, etc., por ejemplo, 
piezas de revolución y piezas prismáticas, etc.

Por lo que para estos tipos de objetos se tiene cuatro formas de reconstrucción y son las siguientes:

a. Reconstrucción mediante superficies automáticas.

b. Reconstrucción mediante modelado sólido.

c. Reconstrucción mediante detección de superficies.

d. Reconstrucción con la ayuda de instrumentos de medición dimensionales.

Para el caso de aplicación realizada se utiliza la siguiente reconstrucción:

- Reconstrucción mediante superficies automáticas: Este tipo de reconstrucción se utiliza para objetos principalmente de tipo orgánico, y lo que hace el software GeomagicDesign X con la herramienta "AUTOSURFACE", es crear una superficie adaptando la misma a la malla de información que fue obtenida por el escáner y de esta manera se pasa de la malla a una superficie a ser utilizada para realizar otras operaciones posteriores. La aproximación de la superficie creada es bastante cercana con respecto a la información del escaneo (ver gráfico 12).

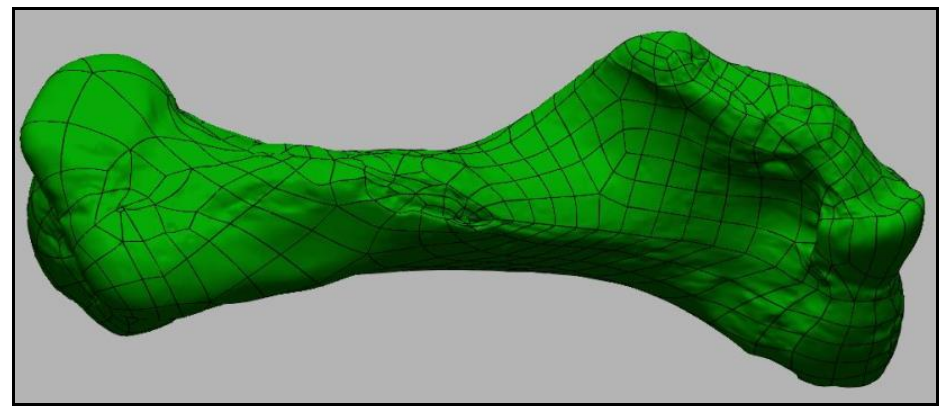

Figura 12. Fósil reconstruido digitalmente.

Fuente: Autores, 2019. 
Revista Arbitrada Interdisciplinaria KOINONIA

Año V. Vol V. Nº. Enero - Junio 2020

Hecho el depósito de Ley: FA2016000010

ISSN: 2542-3088

FUNDACIÓN KOINONIA (F.K). Santa Ana de Coro. Venezuela.

Javier Valverde-Bastidas; Mario Cesén-Arteaqa; Edgar Sarmiento-Boria

Finalmente se obtiene con los pasos anteriores la reconstrucción del fósil de manera digital en el software GeomagicDesign X el cual permite exportar esta información a una extensión de archivo estándar conocida como STL, la misma que posteriormente permite ser utilizada para realizar procesos de prototipado rápido o impresión 3D.

\section{Procedimiento para validar la metodología implementada}

Consiste en comparar los datos de la malla escaneada con el sólido o superficie reconstruida, con lo que se determina que tan aproximado se hizo la reconstrucción con respecto al objeto original, por lo que es en este punto donde se comprueba que tan precisa se realizó la reconstrucción del objeto, para lo cual se tiene la siguiente herramienta:

\section{Accuracy Analyzer ${ }^{\mathrm{TM}}$ en Geomagic Design X}

Esta herramienta permite al usuario comprobar la precisión de su pieza a lo largo de todo el proceso. El cuerpo CAD puede comparar los datos escaneados básicos con sus desviaciones visualizadas en un mapa de color. También aquí se pueden encontrar diferentes formas para visualizar la calidad y continuidad de las superficies. Adicionalmente, se pueden analizar desviaciones entre mallas. Una vez que se aplican las desviaciones, aparecerá una barra de colores para controlar los valores de tolerancia, adicionalmente se pueden tener datos numéricos específicos colocando el puntero del mouse sobre el punto o zona que se desea obtener el valor.

Para poder realizar este análisis se debe tener activada la visualización tanto de la malla como también del sólido o superficie reconstruida y a continuación se siguen los siguientes pasos: 
- Se debe seleccionar la herramienta "AccuracyAnalyzer(TM)" que se encuentra en la parte derecha de la pantalla del software GeomagicDesign X.

- Luego se selecciona en "Type", la opción de "DeviationforBody", con lo que el software empieza a cargar el análisis.

- Luego se coloca un valor de tolerancia de referencia al que se deseaba llegar en la reconstrucción, para que de esta manera el software muestre resultados a partir de ese valor, esto también tiene que ver con las distintas tonalidades que se van a visualizar en el objeto analizado.

- Esta herramienta se puede utilizar, a medida que se van realizando las tareas de reconstrucción para verificar que las mismas se están aproximando adecuadamente a la malla de escaneo; o también se puede utilizar esta herramienta para verificar el sólido o superficie reconstruida al final de todo el procedimiento.

- Finalmente se tiene como resultado que el objeto presenta una gama de colores, que pueden ser modificados (ver gráfico 15), pero normalmente se maneja de esa manera y que tienen el siguiente significado:

- Verde: Señala lugares donde existe mayor aproximación entre el sólido o superficie reconstruido y la información del escaneo de acuerdo a la tolerancia de referencia establecida.

- Rojo: Significa que existe una desviación positiva de la reconstrucción con respecto al malla., es decir en esas partes existe un exceso de material. La tonalidad de los colores puede ser: amarillo, anaranjado y rojo, siendo el último, el de mayores valores de desviaciones existentes.

- Azul: Significa que existe una desviación negativa de la reconstrucción con respecto al malla., es decir es esas partes existe una falta de material. La tonalidad de los colores puede ser: cian, celeste y azul, siendo el último, el 
de mayores valores de desviaciones existentes.

\section{Procedimiento del prototipado del fósil}

En el caso del prototipo rápido, se utiliza la Estación de prototipado rápido "Roland MDX40A", pero primero se debe utilizar el software "SRP Player" (ver figura 13), que es el compatible con el equipo al cual se le carga el archivo (.stl) obtenido anteriormente. Luego se configura en el software los siguientes pasos:

1. Tamaño y orientación del modelo.

2. Tipo de fresado.

3. Creación ruta de la herramienta.

4. Vista preliminar de resultados.

5. Luego configurar la máquina, tanto en el controlador del equipo (software VPanel), como también de forma física en la estación (colocación del material, colocación de herramientas necesarias, cero de referencia, etc.)

6. Realizar el corte.

7.

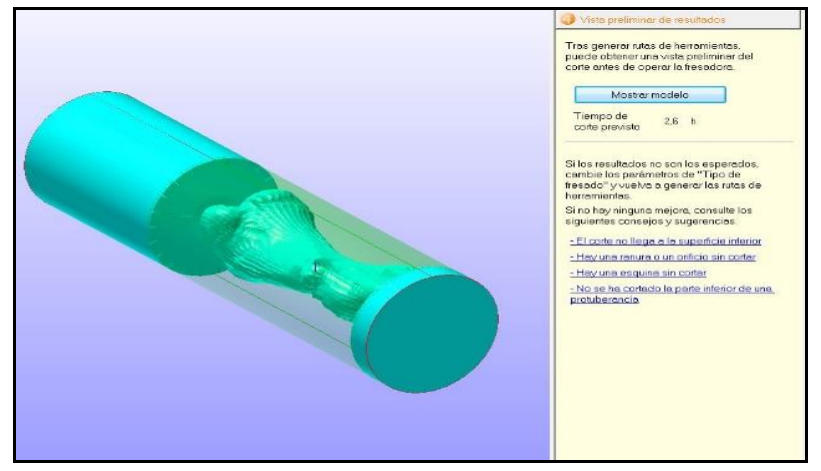

Figura 13. Software SRP Player de la estación de prototipado.

Fuente:Autores, 2019

El prototipo del fósil restaurado se muestra a continuación: 
Revista Arbitrada Interdisciplinaria KOINONIA

Año V. Vol V. Nº. Enero - Junio 2020

Hecho el depósito de Ley: FA2016000010

ISSN: 2542-3088

FUNDACIÓN KOINONIA (F.K). Santa Ana de Coro. Venezuela.

Javier Valverde-Bastidas; Mario Cesén-Arteaqa; Edgar Sarmiento-Boria

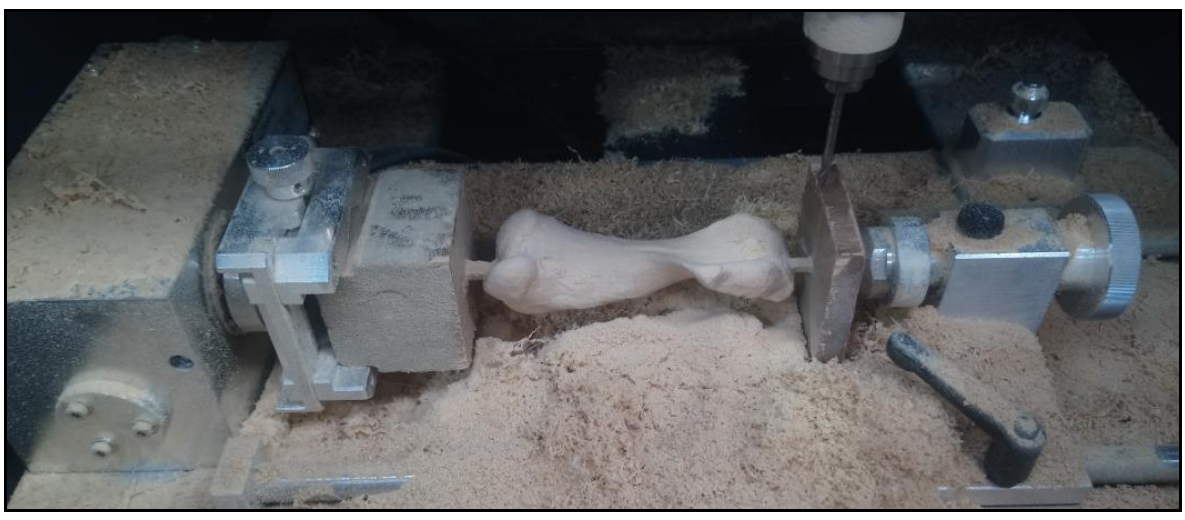

Figura 14. Fósil construido en la estación de prototipado CAM.

Fuente:Autores, 2019

\section{RESULTADOS}

\section{Validación realizada}

Para el caso de la aplicación desarrollada con la metodología indicada anteriormente se realizó validaciones mediante software con la herramienta "AccuracyAnalyzer TM" en el software GeomagicDesign, las mismas que fueron consideradas con un valor de tolerancia de referencia de $0,1 \mathrm{~mm}$ para poder realizar el análisis de resultados y se obtuvo la siguiente información:

\section{Tabla1}

Resultados del análisis de desviación del fósil.

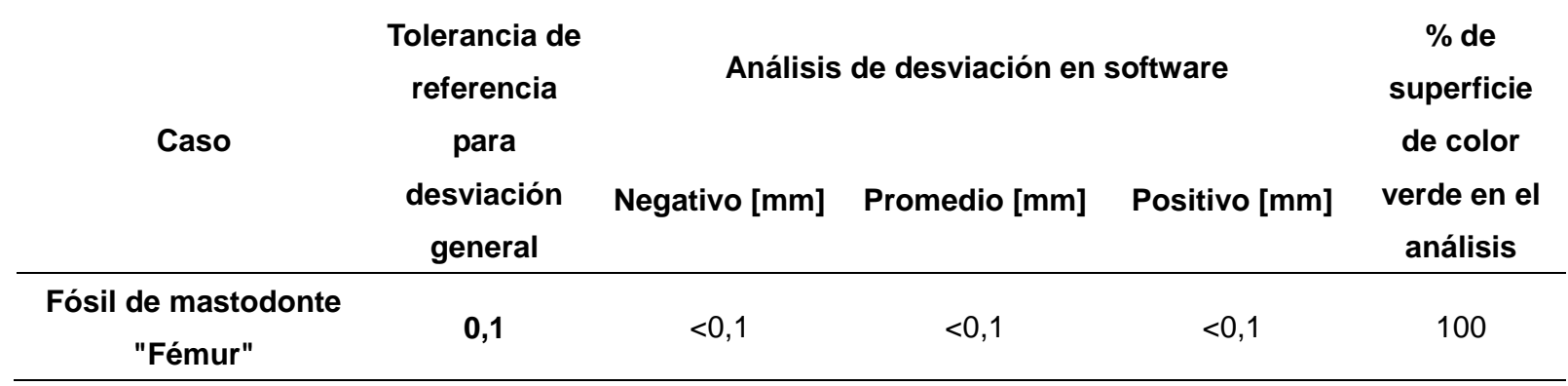

Fuente:Autores, 2019

Además se tiene un cien por ciento de porcentaje de superficie de color verde lo que 
significa que visualmente se puede evidenciar que la desviación de medidas entre la reconstrucción 3D con respecto a la malla obtenida en el escaneo 3D es menor a 0,1 milímetros, tal como se muestra en el siguiente gráfico:

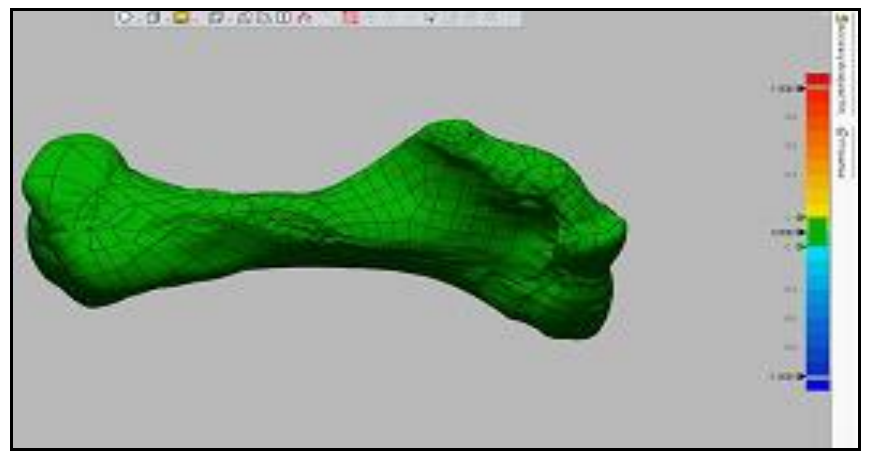

Figura 15. Análisis de desviación del fósil.

Fuente:Autores, 2019

\section{DISCUSIÓN}

De acuerdo a los resultados obtenidos de cada una de las fases de aplicación desarrollada mediante el escaneo 3D y las validaciones realizadas, se puede mencionar que al escanear un objeto, se deben considerar algunas metodologías para poder obtener de la mejor manera el resultado de la información de escaneado, el post-tratamiento y la reconstrucción del objeto. Para el caso de aplicación desarrollada (reconstrucción de un fósil), se obtuvo la información final satisfactoriamente como se muestra en la figura 15, donde se puede verificar con color verde toda la superficie del fósil y además en la tabla 1, se tiene valores de desviación menores al 0,1 milímetros. Todo esto fue gracias a que se siguió una correcta metodología, ya que se tienen datos reales y muy aproximados del fósil original, ya sea de forma digital y también de forma física mediante el prototipado del mismo.

Es importante considerar la resolución de escaneo, si se quiere mayor cantidad de 
información de los detalles y de forma precisa, se tiene que trabajar con resoluciones más bajas. Del trabajo realizado se puede establecer que la resolución y velocidad del obturador del escáner utilizado, estuvieron correctamente seleccionados, se obtuvo la información adecuada y sin que falte información o detalles importantes del fósil

Cuando el fósil es muy grande la información de escaneo es demasiado alta y muy pesada y esto se debe tomar en cuenta al momento de guardar la información, pero principalmente, al realizar el post-tratamiento y la reconstrucción. También fue importante escanear todas las dos partes que se tenían del fósil para luego realizar la fusión, restauración y reconstrucción del mismo.

En el post-tratamiento se realizó una fase de limpieza, alineación, llenado de agujeros, afinado y mejoras en la malla, de tal manera que no se tenga errores en la reconstrucción.

Se utilizaron las herramientas más adecuadas para poder obtener el objeto reconstruido tridimensionalmente, ya que, pueden existir varias maneras de realizar estas operaciones de reconstrucción y esto se puede verificar al momento de la realización del análisis de desviación.

\section{CONCLUSIÓN}

Se establecieron metodologías adecuadas para este tipo de aplicación mediante escaneado 3D, obteniéndose un fósil reconstruido muy aproximado al original, con lo que en base a esta información se pueden desarrollar otros tipos de restauraciones.

En el presente trabajo se utilizaron tecnologías modernas de escaneado 3D y prototipado rápido, que permitieron obtener un objeto muy parecido al fósil utilizado, además que se realizó una integración eficiente de los equipos utilizados.

Para un adecuado análisis de desviación, se debe establecer un valor de tolerancia de desviación es de referencia para la reconstrucción 3D adecuado y para esto es 
Revista Arbitrada Interdisciplinaria KOINONIA

Año V. Vol V. Nº. Enero - Junio 2020

Hecho el depósito de Ley: FA2016000010

ISSN: 2542-3088

FUNDACIÓN KOINONIA (F.K). Santa Ana de Coro. Venezuela.

Javier Valverde-Bastidas; Mario Cesén-Arteaqa; Edqar Sarmiento-Boria

importante considerar la naturaleza del objeto de referencia, ya que si no se logra al primer desarrollo o intento, se puede encontrar otras herramientas que me permitan lograr resultados más aproximados.

El tiempo total que se emplea para realizar todas las operaciones desde el escaneado hasta la obtención del producto final, depende de algunos factores inherentes a los procedimientos señalados que se deben seguir, pero sobre todo depende de qué tan complejo es el objeto de referencia a trabajar, además del tamaño del mismo, ya que si se tiene un gran tamaño, se maneja para todos los procedimientos con archivos de gran tamaño de memoria, para lo cual se necesita un recurso informático mayor.

\section{FINANCIAMIENTO}

No monetario

\section{AGRADECIMIENTO}

Un agradecimiento especial al Laboratorio de Investigación, Desarrollo e Innovación en Nuevas Tecnologías de Diseño, Manufactura y Producción (LABINTECDIMP) de la Facultad de Ingeniería Mecánica de la Escuela Politécnica Nacional por la apertura para el desarrollo del presente trabajo, tanto con el personal y también con los equipos que se utilizaron del mismo. 
Revista Arbitrada Interdisciplinaria KOINONIA

Año V. Vol V. Nº. Enero - Junio 2020

Hecho el depósito de Ley: FA2016000010

ISSN: 2542-3088

FUNDACIÓN KOINONIA (F.K). Santa Ana de Coro. Venezuela.

Javier Valverde-Bastidas; Mario Cesén-Arteaqa; Edgar Sarmiento-Boria

\section{REFERENCIAS CONSULTADAS}

Accurex (2017). Software Geomagic Design. [Geomagic Design X Software]. Recuperado de https://www.accurexmeasure.com/

Ali, N. S. (2005). Reverse Engineering of automotive parts applying laser scanning and structured light techniques. [Ingeniería inversa de piezas automotrices aplicando escaneo láser y técnicas de luz estructurada]. Project in Lieu of Thesis presented for the Masters of Science Degree, The University of Tennessee, Knoxville.

Boboulos, M. A. (2010). CAD-CAM \& rapid prototyping application evaluation. Bookboon. [CAD-CAM y evaluación de aplicaciones del prototipado rápido. Bookboon].

Borja, V. (1997). Redesign supported by data models with particular reference. [Rediseño soportado por modelos de datos con referencia particular].

Ceniceros, M. M. (2017). Puesta en marcha de un escáner 3D y aplicación de ingeniería inversa y fabricación aditiva. [Implementing of a 3D scanner and application of reverse engineering and additive manufacturing].

Fernández Zúñiga, E. J., Julio, C., \& Marcela, C. (2014). Diseño e implementación de un escáner 3D para prototipado y modelado geométrico de objetos. [Design and implementation of a 3D scanner for prototyping and geometric modeling of objects].

García, A., Ruiz, J., Jiménez, L., Reyes, L., Luna, G., Ontiveros, S. \& Carrillo, E. (2008). Clasificación de programas y Modelos de la Ingeniería Inversa: Aplicaciones a un caso de estudio. [Classification of reverse engineering programs and models: Applications to a case study]. SOMIM2008. Puebla, México.

Martínez, A. B., \& Salcedo, A. F. C. (2012). Escáner 3d para control de calidad de piezas metalúrgicas. [ $3 \mathrm{~d}$ scanner for quality control of metallurgical parts].

Morillo, M. A. (2015). Digitalización 3D con escáner de luz estructurada aplicada al área de la gestión de calidad y la conservación del patrimonio histórico-artístico. [3D scanning with a structured light scanner applied to the area of quality management and conservation of the historical-artistic heritage].

Radhakrishnan, P., Subramanyan, S., \&Raju, V. (2008). CAD/CAM/CIM. New Age International. [CAD / CAM / CIM. New Age International]. 
Revista Arbitrada Interdisciplinaria KOINONIA

Año V. Vol V. Nº. Enero - Junio 2020

Hecho el depósito de Ley: FA2016000010

ISSN: 2542-3088

FUNDACIÓN KOINONIA (F.K). Santa Ana de Coro. Venezuela.

Javier Valverde-Bastidas; Mario Cesén-Arteaqa; Edgar Sarmiento-Boria

Raja, V., \& Fernandes, K. J. (Eds.). (2007). Reverse engineering: an industrial perspective. [Ingeniería inversa: una perspectiva industrial]. SpringerScience\& Business Media.

Romeral Pérez, F. (2014). Flujo de trabajo en proyectos de modelización 3D con equipos láser escáner. [Workflow in 3D modeling projects with laser scanner equipment].

Sánchez (2017) ¿Cómo elegir un escáner 3D? [How to choose a 3D scanner?]. Recuperado de https://n9.cl/dvy8

Sebastián, J. M. T. (2013). Escaneado en 3D y prototipado de piezas Patrimonio Arqueológico. [3D scanning and prototyping of pieces Archaeological Heritage]. Iberia. Revista de la Antigüedad, 8, 135-158.

Roland DGA (2019). Fresado automatizado 3D. [Automated 3D milling]. Recuperado de https://n9.cl//kbut

Valverde Bastidas, J. G. (2019). Desarrollo de metodologías enfocadas a aplicaciones de ingeniería inversa para reproducir objetos mediante escaneado $3 D$, sistemas CAD/CAM y prototipado rápido (Master's thesis, Quito, 2019.). [Development of methodologies focused on reverse engineering applications to execute objects using 3D scanning, CAD / CAM systems and rapid prototyping (Tesis de maestría, Quito, 2019.)]. Recuperado de https://n9.cl/0nv2

Wang, W. (2010). Reverse engineering: Technology of reinvention. [Ingeniería inversa: tecnología de reinvención]. CrcPress. 